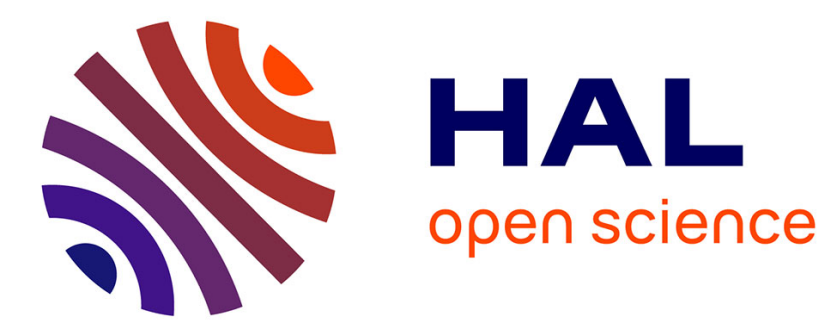

\title{
Inverse problem stability of a continuous-in-time financial model
}

Tarik Chakkour

\section{To cite this version:}

Tarik Chakkour. Inverse problem stability of a continuous-in-time financial model. Acta Mathematica Scientia, 2019, 39 (5), pp.1423-1439. 10.1007/s10473-019-0519-5 . hal-02618047

\section{HAL Id: hal-02618047 https://hal.inrae.fr/hal-02618047}

Submitted on 25 May 2020

HAL is a multi-disciplinary open access archive for the deposit and dissemination of scientific research documents, whether they are published or not. The documents may come from teaching and research institutions in France or abroad, or from public or private research centers.
L'archive ouverte pluridisciplinaire HAL, est destinée au dépôt et à la diffusion de documents scientifiques de niveau recherche, publiés ou non, émanant des établissements d'enseignement et de recherche français ou étrangers, des laboratoires publics ou privés. 


\title{
Inverse problem stability of a continuous-in-time financial model
}

\author{
Tarik Chakkour
}

\author{
Piaf INRA, F-63000 Clermont-Ferrand, France
}

September 23, 2019

\begin{abstract}
In this work, we study the inverse problem stability of the continuous-in-time model which is designed to be used for the finances of public institutions. We discuss this study with determining the Loan Measure from Algebraic Spending Measure in Radon measure space $\mathcal{M}\left(\left[t_{\mathrm{I}}, \Theta_{\text {max }}\right]\right)$, and in Hilbert space $\mathbb{L}^{2}\left(\left[t_{\mathrm{I}}, \Theta_{\max }\right]\right)$ when they are density measures. For this inverse problem we prove the uniqueness theorem, obtain a procedure for constructing the solution and provide necessary and sufficient conditions for the solvability of the inverse problem in $\mathbb{L}^{2}\left(\left[t_{\mathrm{I}}, \Theta_{\max }\right]\right)$.
\end{abstract}

Keywords: Inverse problem; stability; mathematical model; Fredholm operator.

\section{Introduction}

In the last two decades, the theory and practice of inverse problems have been developed in many scientific domains. Consequently, it is rapidly growing, if not exploding. Moreover, document [1] shows how much researchers contribute to this field. Many inverse problems arising in scientific domains present numerical instability: the noise affecting the data may produce arbitrarily large errors in the solutions. In other words, these problems are ill-posed in the sense of Hadamard. The concept of ill-posedness was introduced by HADAMARD [11] in the field of partial differential equations. We mention the book on the mathematics of ill-posed problems by TIKHONOV and ARSENIN [13].

We bluild in previous works $[10,6]$ the continuous-in-time model which is based on using the mathematical tools such convolution and integration. Indeed, this model uses measures over time interval to describe loan scheme, reimbursement scheme and interest payment scheme. The model contains some financial quantities. For instance, the Repayment Pattern Measure $\tilde{\gamma}$ is a non-negative measure with total mass which equals 1, the Algebraic Spending Measure $\tilde{\sigma}$ is defined such that the difference between spendings and incomes required to satisfy the current needs between times $t_{1}$ and $t_{2}$ is:

$$
\int_{t_{1}}^{t_{2}} \tilde{\sigma}
$$

and the Loan Measure $\tilde{\kappa}_{E}$ is defined such that the amount borrowed between times $t_{1}$ and $t_{2}$ is:

$$
\int_{t_{1}}^{t_{2}} \tilde{\kappa}_{E}
$$

When measure $\tilde{\gamma}$ is absolutely continuous with respect to the Lebesgue measure $d t$. This means that it read $\gamma(t) d t$, where $t$ is the variable in $\mathbb{R}$. The work [10] proposes the resolution of the inverse problem over the space of square-integrable functions when density $\gamma$ is equal to $\frac{1}{\Theta_{\gamma}}$ over time period $\left[0, \Theta_{\gamma}\right]$ and to 0 elsewhere. In papers $[7,5]$, we use a mathematical framework to discuss an inverse problem of determining the Loan Measure $\tilde{\kappa}_{E}$ from Algebraic Spending Measure $\tilde{\sigma}$. This inverse problem is used in [10] on simplified examples in order to show its capability to be used to forecast a financial strategy. In [4] we investigate the properties of operators and we discuss an inverse problem in Schwartz space that 
we prove the uniqueness theorem.

This paper is concerned with continuity and compactness of some operators involved in the model. We refer papers $[8,3,2]$ that treat this topic. In the paper [8], M. D. Sen investigates the system stability and the compactness of the operators describing the solution trajectories. M. Cecchi, M. Furi and M. Martin study in [3] continuity and compactness of some nonlinear operators under the topology of space. In addition, R. I. Becker has discussed in [2] the compactness of certain integral operators under the topology in $\mathbb{L}^{2}$. In the sequel, we will show in this paper that these operators are continuous and are not compact on $\mathbb{L}^{2}$.

We show in this paper the general results of this inverse problem for any density $\gamma$. We show the stability of the inverse problem. We continue to extend some results of this inverse problem in measure space with proving its stability. In other words, we describe a more complete numerical study for the inverse problem. The main result of this paper is the existence and uniqueness of solutions for the system modeling the financial multiyear planning. This result ensures the mathematical well-posedness under the balanced equation assumption.

The organization of the rest of this paper is as follows. Section 2 describes the inverse problem of the model in $\mathbb{L}^{2}\left(\left[t_{\mathrm{I}}, \Theta_{\max }\right]\right)$. Section 3 shows the inverse problem of the model in $\mathcal{M}\left(\left[t_{\mathrm{I}}, \Theta_{\max }\right]\right)$.

\section{Inverse problem of the model in $\mathbb{L}^{2}\left(\left[t_{\mathrm{I}}, \Theta_{\max }\right]\right)$}

Denoting $\mathbb{L}^{2}\left(\left[t_{I}, \Theta_{\max }\right]\right)$ the space of square-integrable functions over $\mathbb{R}$ having their support in $\left[t_{\mathrm{I}}, \Theta_{\max }\right]$ and denoting $\mathbb{L}^{2}\left(\left[0, \Theta_{\gamma}\right]\right)$ the space of square-integrable functions over $\mathbb{R}$ having their support in $\left[0, \Theta_{\gamma}\right]$. We state the Repayment Pattern Density $\gamma$ as follows:

$$
\gamma \in \mathbb{L}^{2}\left(\left[0, \Theta_{\gamma}\right]\right),
$$

where $\Theta_{\gamma}$ is a positive number such that:

$$
\Theta_{\gamma}<\Theta_{\max }-t_{\mathrm{I}}
$$

We justify relation (3) because the support of convolution of two compactly supported densities $\kappa_{E}$ in $\left[t_{\mathrm{I}}, \Theta_{\max }-\Theta_{\gamma}\right]$ and $\gamma$ in $\left[0, \Theta_{\gamma}\right]$ is included in $\left[t_{\mathrm{I}}, \Theta_{\max }\right]$.

Lemma 2.1. Linear operator $\mathcal{L}$ acting on Loan Density $\kappa_{E} \in \mathbb{L}^{2}\left(\left[t_{\mathrm{I}}, \Theta_{\max }-\Theta_{\gamma}\right]\right)$ defined as:

$$
\mathcal{L}\left[\kappa_{E}\right](t)=\kappa_{E}(t)-\left(\kappa_{E} \star \gamma\right)(t)-\alpha \int_{t_{\mathrm{I}}}^{t}\left(\kappa_{E}-\kappa_{E} \star \gamma\right)(s) d s,
$$

is continuous and is not compact operator on $\mathbb{L}^{2}\left(\left[t_{\mathrm{I}}, \Theta_{\max }\right]\right)$. Here, the operator $\star$ involved in (5) is the convolution operator.

Proof. In the first place, we will show that operator $\mathcal{L}$ is well defined and continuous. That why, we will show that for any density $\kappa_{E}$ in $\mathbb{L}^{2}\left(\left[t_{\mathbb{I}}, \Theta_{\max }-\Theta_{\gamma}\right]\right)$, function $\mathcal{L}\left[\kappa_{E}\right]$ is square-integrable function over $\mathbb{R}$ having its support in $\left[t_{\mathrm{I}}, \Theta_{\max }\right]$. Formally, we will prove:

$$
\left\|\mathcal{L}\left[\kappa_{E}\right]\right\|_{\mathbb{L}^{2}\left(\left[t_{\mathrm{I}}, \Theta_{\max }\right]\right)} \leq\left(1+|\alpha| \times\left(\Theta_{\max }-t_{\mathrm{I}}\right)\right) \times\left(1+\|\gamma\|_{\mathbb{L}^{1}\left(\left[0, \Theta_{\gamma}\right]\right)}\right) \times\left\|\kappa_{E}\right\|_{\mathbb{L}^{2}\left(\left[t_{\mathrm{I}}, \Theta_{\max }-\Theta_{\gamma}\right]\right)} .
$$

Taking norm $\mathbb{L}^{2}\left(\left[t_{I}, \Theta_{\max }\right]\right)$ and applying triangle inequality to definition (5) of operator $\mathcal{L}$, we obtain the following inequality:

$$
\left\|\mathcal{L}\left[\kappa_{E}\right]\right\|_{\mathbb{L}^{2}\left(\left[t_{1}, \Theta_{\max }\right]\right)} \leq\left\|\kappa_{E}-\kappa_{E} \star \gamma\right\|_{\mathbb{L}^{2}\left(\left[t_{\mathrm{I}}, \Theta_{\max }\right]\right)}+|\alpha| \times C_{E},
$$


where $C_{E}$ is defined and is increased as follows:

$$
\begin{aligned}
C_{E} & =\left\|\int_{t_{\mathrm{I}}}^{t} \kappa_{E}(s)-\kappa_{E} \star \gamma(s) d s\right\|_{\mathbb{L}^{2}\left(\left[t_{\mathrm{I}}, \Theta_{\max }\right]\right)}, \\
& =\sqrt{\int_{t_{\mathrm{I}}}^{\Theta_{\max }}\left(\int_{t_{\mathrm{I}}}^{t} \kappa_{E}(s)-\kappa_{E} \star \gamma(s) d s\right)^{2} d t}, \\
& \leq \sqrt{\Theta_{\max }-t_{\mathrm{I}}} \times\left\|\kappa_{E_{2}}-\kappa_{E} \star \gamma\right\|_{\mathbb{L}^{1}\left(\left[t_{\mathrm{I}}, \Theta_{\max }\right]\right)} .
\end{aligned}
$$

We use Cauchy-Schwarz inequality to obtain:

$$
\begin{aligned}
\left\|\kappa_{E}-\kappa_{E} \star \gamma\right\|_{\mathbb{L}^{1}\left(\left[t_{\mathrm{I}}, \Theta_{\max }\right]\right)} & =\int_{t_{\mathrm{I}}}^{\Theta_{\max }} 1 \times\left|\kappa_{E}(s)-\kappa_{E} \star \gamma(s)\right| d s, \\
& \leq \sqrt{\int_{t_{\mathrm{I}}}^{\Theta_{\max }} 1^{2} d s} \\
& \times \sqrt{\int_{t_{\mathrm{I}}}^{\Theta_{\max }}\left(\kappa_{E}(s)-\left(\kappa_{E} \star \gamma\right)(s)\right)^{2}} d s \\
& \leq \sqrt{\Theta_{\max }-t_{\mathrm{I}}} \times\left\|\kappa_{E}-\kappa_{E} \star \gamma\right\|_{\mathbb{L}^{2}\left(\left[t_{\mathrm{I}}, \Theta_{\max }\right]\right)}
\end{aligned}
$$

Thanks to properties (8) and (9), we get:

$$
C_{E} \leq\left(\Theta_{\max }-t_{\mathrm{I}}\right) \times\left\|\kappa_{E}-\kappa_{E} \star \gamma\right\|_{\mathbb{L}^{2}\left(\left[t_{\mathrm{I}}, \Theta_{\max }\right]\right)} .
$$

From this and according to (7), we get:

$$
\left\|\mathcal{L}\left[\kappa_{E}\right]\right\|_{\mathbb{L}^{2}\left(\left[t_{\mathrm{I}}, \Theta_{\max }\right]\right)} \leq\left(1+|\alpha| \times\left(\Theta_{\max }-t_{\mathrm{I}}\right)\right) \times\left\|\kappa_{E}-\kappa_{E} \star \gamma\right\|_{\mathbb{L}^{2}\left(\left[t_{\mathrm{I}}, \Theta_{\max }\right]\right)} .
$$

The triangle and the Young's inequalities implie that:

$$
\begin{aligned}
& \left\|\kappa_{E}-\kappa_{E} \star \gamma\right\|_{\mathbb{L}^{2}\left(\left[t_{\mathrm{I}}, \Theta_{\max }\right]\right)} \leq\left\|\kappa_{E}\right\|_{\mathbb{L}^{2}\left(\left[t_{\mathrm{I}}, \Theta_{\max }-\Theta_{\gamma}\right]\right)}+\left\|\kappa_{E} \star \gamma\right\|_{\mathbb{L}^{2}\left(\left[t_{\mathrm{I}}, \Theta_{\max }\right]\right)}, \\
& \leq\left\|\kappa_{E}\right\|_{\mathbb{L}^{2}\left(\left[t_{\mathrm{I}}, \Theta_{\max }-\Theta_{\gamma}\right]\right)}+\left\|\kappa_{E}\right\|_{\mathbb{L}^{2}\left(\left[t_{\mathrm{I}}, \Theta_{\max }-\Theta_{\gamma}\right]\right)}\|\gamma\|_{\mathbb{L}^{1}\left(\left[0, \Theta_{\gamma}\right]\right)}, \\
& \leq\left(1+\|\gamma\|_{\mathbb{L}^{1}\left(\left[0, \Theta_{\gamma}\right]\right)}\right) \times\left\|\kappa_{E}\right\|_{\mathbb{L}^{2}\left(\left[t_{\mathrm{I}}, \Theta_{\max }-\Theta_{\gamma}\right]\right)} .
\end{aligned}
$$

Inequality (12) yields with (11) to achieve the proof of inequality (6). From this, $\mathcal{L}$ is a continuous map from $\mathbb{L}^{2}\left(\left[t_{\mathrm{I}}, \Theta_{\max }-\Theta_{\gamma}\right]\right)$ to $\mathbb{L}^{2}\left(\left[t_{\mathrm{I}}, \Theta_{\max }\right]\right)$. In the second place, decomposing operator $\mathcal{L}$ in following form:

$$
\mathcal{L}\left[\kappa_{E}\right]+\tilde{\mathcal{V}}\left[\kappa_{E}\right]=I d_{\mathbb{L}^{2}\left(\left[t_{I}, \Theta_{\max }-\Theta_{\gamma}\right] \rightarrow \mathbb{L}^{2}\left(\left[t_{I}, \Theta_{\max }\right]\right)\right.},
$$

where operator $\tilde{\mathcal{V}}: \mathbb{L}^{2}\left(\left[t_{\mathrm{I}}, \Theta_{\max }-\Theta_{\gamma}\right]\right) \rightarrow \mathbb{L}^{2}\left(\left[t_{\mathrm{I}}, \Theta_{\max }\right]\right)$ is defined by the operator:

$$
\tilde{\mathcal{V}}\left[\kappa_{E}\right](t)=\kappa_{E} \star \gamma(t)+\alpha \int_{t_{\mathrm{I}}}^{t}\left(\kappa_{E}-\kappa_{E} \star \gamma\right)(s) d s .
$$

The target of decomposition (13) is to show with using absurd reasoning that $\mathcal{L}$ is not compact. In order to emerge a contradiction, we will show that $\tilde{\mathcal{V}}$ is compact on $\mathbb{L}^{2}\left(\left[t_{\mathrm{I}}, \Theta_{\text {max }}\right]\right)$. Indeed, with showing that $\tilde{\mathcal{V}}$ it will be, we obtain that $I d_{\mathbb{L}^{2}\left(\left[t_{I}, \Theta_{\max }-\Theta_{\gamma}\right]\right) \rightarrow \mathbb{L}^{2}\left(\left[t_{I}, \Theta_{\max }\right]\right)}$ is compact, which is not possible due to its no compactness. We define the set 


$$
B_{r}=\left\{e \in \mathbb{L}^{2}\left(\left[t_{\mathrm{I}}, \Theta_{\max }-\Theta_{\gamma}\right]\right):\|e\|_{\mathbb{L}^{2}\left(\left[t_{\mathrm{I}}, \Theta_{\max }-\Theta_{\gamma}\right]\right)}<r\right\},
$$

as the open ball of radius $r$ and origine center on $L^{\infty}\left(\left[t_{\mathrm{I}}, \Theta_{\max }\right]\right)$ and the set

$$
B_{r}^{\prime}=\left\{e \in \mathcal{C}_{c}\left(\left[t_{\mathrm{I}}, \Theta_{\max }\right]\right):\|e\|_{L^{\infty}\left(\left[t_{\mathrm{I}}, \Theta_{\max }\right]\right)}<r\right\},
$$

as the open ball of radius $r$ and origine center on $L^{\infty}\left(\left[t_{\mathrm{I}}, \Theta_{\max }\right]\right)$. Here $\mathcal{C}_{c}\left(\left[t_{\mathrm{I}}, \Theta_{\max }\right]\right)$ is a continuous functions space defined over a time interval $\left[t_{\mathrm{I}}, \Theta_{\max }\right]$ with usual norm is:

$$
\|\psi\|_{L^{\infty}\left(\left[t_{\mathrm{I}}, \Theta_{\max }\right]\right)}=\sup _{t \in\left[t_{\mathrm{I}}, \Theta_{\max }\right]}\{|\psi(t)|\} .
$$

Denoting $\overline{B_{r}}$ and $\overline{B_{r}^{\prime}}$ respectively the closed balls of open balls $B_{r}$ and $B_{r}^{\prime}$. In order to show that operator $\mathcal{V}: \mathbb{L}^{2}\left(\left[t_{\mathrm{I}}, \Theta_{\max }-\Theta_{\gamma}\right]\right) \rightarrow \mathcal{C}_{c}\left(\left[t_{\mathrm{I}}, \Theta_{\max }\right]\right)$ defined as the considered operator $\tilde{\mathcal{V}}$ from $\mathbb{L}^{2}\left(\left[t_{\mathrm{I}}, \Theta_{\max }-\Theta_{\gamma}\right]\right)$ to $\mathcal{C}_{c}\left(\left[t_{\mathrm{I}}, \Theta_{\max }\right]\right)$ is compact, we will show that the image of the open unit ball $B_{1}$ under $\mathcal{V}$

$$
E_{1}=\mathcal{V}\left(B_{1}\right)
$$

is relatively compact in $\mathcal{C}_{c}\left(\left[t_{\mathrm{I}}, \Theta_{\max }\right]\right)$. Since bounded and equicontinuous sets of $\mathcal{C}_{c}\left(\left[t_{\mathrm{I}}, \Theta_{\max }\right]\right)$ are relatively compacts, we verify a compactness criteria of $E_{1}$ defined by (18) with using Ascoli theorem. Indeed, showing this criteria consists in showing that $E_{1}$ is a bounded subset of $\mathcal{C}_{c}\left(\left[t_{\mathrm{I}}, \Theta_{\max }\right]\right)$. First, applying triangle inequality to operator $\mathcal{V}$ to obtain the following inequality:

$$
\begin{aligned}
\left|\mathcal{V}\left[\kappa_{E}\right](t)\right| & \leq\left|\kappa_{E} \star \gamma(t)\right|+|\alpha|\left|\int_{t_{\mathrm{I}}}^{t}\left(\kappa_{E}-\kappa_{E} \star \gamma\right)(s) d s\right|, \\
& \leq\left|\kappa_{E} \star \gamma(t)\right|+|\alpha| \int_{t_{\mathrm{I}}}^{t}\left|\left(\kappa_{E}-\kappa_{E} \star \gamma\right)(s)\right| d s, \\
& \leq\left|\kappa_{E} \star \gamma(t)\right|+|\alpha||| \kappa_{E}-\kappa_{E} \star \gamma \|_{\mathbb{L}^{1}\left(\left[t_{\mathrm{I}}, \Theta_{\max }\right]\right) .}
\end{aligned}
$$

Next, we use Cauchy-Schwarz inequality to obtain:

$$
\begin{aligned}
\left|\kappa_{E} \star \gamma(t)\right| & =\left|\int_{t_{\mathrm{I}}}^{t} \gamma(t-s) \kappa_{E}(s) d s\right|, \\
& \leq \int_{t_{\mathrm{I}}}^{\Theta_{\max }}\left|\gamma(t-s) \kappa_{E}(s)\right| d s, \\
& \leq \sqrt{\int_{t_{\mathrm{I}}}^{\Theta_{\max }}(\gamma(t-s))^{2}} d s \sqrt{\int_{t_{\mathrm{I}}}^{\Theta_{\max }}\left(\kappa_{E}(s)\right)^{2} d s .}
\end{aligned}
$$

Since densities $\kappa_{E}$ and $\gamma$ are supported respectively in intervals $\left[t_{\mathrm{I}}, \Theta_{\max }-\Theta_{\gamma}\right]$ and $\left[0, \Theta_{\gamma}\right]$, inequality (20) is written in following form:

$$
\left|\kappa_{E} \star \gamma(t)\right| \leq\|\gamma\|_{\mathbb{L}^{2}\left(\left[0, \Theta_{\gamma}\right]\right)}\left\|\kappa_{E}\right\|_{\mathbb{L}^{2}\left(\left[t_{\mathbb{T}}, \Theta_{\max }-\Theta_{\gamma}\right]\right)} .
$$

Then, relations (9), (12), (19) and (21) yield the following inequality:

$$
\left\|\mid \mathcal{V}\left[\kappa_{E}\right]\right\|_{L^{\infty}\left(\left[t_{\mathrm{I}}, \Theta_{\max }\right]\right)} \leq\left(\|\gamma\|_{\mathbb{L}^{2}\left(\left[0, \Theta_{\gamma}\right]\right)}+|\alpha| \sqrt{\Theta_{\max }-t_{\mathrm{I}}} \times\left(1+\|\gamma\|_{\mathbb{L}^{1}\left(\left[0, \Theta_{\gamma}\right]\right)}\right)\right)\left\|\kappa_{E}\right\|_{\mathbb{L}^{2}\left(\left[t_{\mathrm{I}}, \Theta_{\max }-\Theta_{\gamma}\right]\right)}
$$


Since density $\kappa_{E}$ is in unit ball $B_{1}$ and density $\gamma$ satisfies (3), inequality (22) shows that there exists a positive constant $C_{1}$ defined as:

$$
C_{1}=\|\gamma\|_{\mathbb{L}^{2}\left(\left[0, \Theta_{\gamma}\right]\right)}+|\alpha| \sqrt{\Theta_{\max }-t_{\mathrm{I}}} \times\left(1+\|\gamma\|_{\mathbb{L}^{1}\left(\left[0, \Theta_{\gamma}\right]\right)}\right)
$$

such that we have:

$$
\left\|\mid \mathcal{V}\left[\kappa_{E}\right]\right\|_{L^{\infty}\left(\left[t_{\mathrm{I}}, \Theta_{\max }\right]\right)} \leq C_{1}
$$

Inequality (24) means that $E_{1}$ is contained in closed unit ball of center 0 , and radius $C_{1}$, i.e. $E \subset \overline{B_{C_{1}}^{\prime}}$. Consequently, $E$ defined by (18) is a bounded subset of $\mathcal{C}_{c}\left(\left[t_{\mathrm{I}}, \Theta_{\max }\right]\right)$. Now, we will demonstrate that $E_{1}$ defined by (18) is an equicontinuous subset of $\mathcal{C}_{c}\left(\left[t_{\mathrm{I}}, \Theta_{\max }\right]\right)$. Suppose that $\kappa_{E}$ is in unit ball $B_{1}$, we will show:

$$
\forall x, y \in\left[t_{\mathrm{I}}, \Theta_{\max }\right], \forall \epsilon>0, \exists \eta=\eta(\epsilon):|x-y| \leq \eta \Longrightarrow\left|\mathcal{V}\left[\kappa_{E}\right](y)-\mathcal{V}\left[\kappa_{E}\right](x)\right|<\epsilon .
$$

We start by establishing that for any reals $x$ and $y$ in $\left[t_{\mathrm{I}}, \Theta_{\max }\right]$ the following inequality:

$$
\left|\mathcal{V}\left[\kappa_{E}\right](y)-\mathcal{V}\left[\kappa_{E}\right](x)\right| \leq\left|\int_{x}^{y} \gamma(t-s) \kappa_{E}(s) d s\right|+|\alpha|\left|\int_{x}^{y}\left(\kappa_{E}-\kappa_{E} \star \gamma\right)(s) d s\right| .
$$

Applying Cauchy-Schwarz inequality to each right terms of inequality (26), we obtain:

$$
\left|\mathcal{V}\left[\kappa_{E}\right](y)-\mathcal{V}\left[\kappa_{E}\right](x)\right| \leq \sqrt{|y-x|} \times\left(\sqrt{\left|\int_{x}^{y}\left(\gamma(t-s) \kappa_{E}(s)\right)^{2} d s\right|}+|\alpha| \sqrt{\left.\mid \int_{x}^{y}\left(\kappa_{E}-\kappa_{E} \star \gamma\right)(s)\right)^{2} \mid} d s\right)
$$

Furthermore, since $x$ and $y$ are in interval $\left[t_{\mathrm{I}}, \Theta_{\max }\right]$, inequality (27) implies that:

$$
\begin{aligned}
\left|\mathcal{V}\left[\kappa_{E}\right](y)-\mathcal{V}\left[\kappa_{E}\right](x)\right| & \leq \sqrt{|y-x|} \times\left(\sqrt{\int_{t_{\mathrm{I}}}^{\Theta_{\max }}\left(\gamma(t-s) \kappa_{E}(s)\right)^{2}} d s+|\alpha| \sqrt{\left.\int_{t_{\mathrm{I}}}^{\Theta_{\max }}\left(\kappa_{E}-\kappa_{E} \star \gamma\right)(s)\right)^{2}} d s\right) \\
& \leq \sqrt{|y-x|} \times\left(\left\|\kappa_{E} \star \gamma\right\|_{\mathbb{L}^{2}\left(\left[t_{\mathrm{I}}, \Theta_{\max }\right]\right)}+|\alpha|\left\|\kappa_{E}-\kappa_{E} \star \gamma\right\|_{\mathbb{L}^{2}\left(\left[t_{\mathrm{I}}, \Theta_{\max }\right]\right)}\right) .
\end{aligned}
$$

From this and according to (12), we get:

$$
\left|\mathcal{V}\left[\kappa_{E}\right](y)-\mathcal{V}\left[\kappa_{E}\right](x)\right| \leq \sqrt{|y-x|} \times\left(\|\gamma\|_{\mathbb{L}^{1}\left(\left[0, \Theta_{\gamma}\right]\right)}+|\alpha|\left(1+\|\gamma\|_{\mathbb{L}^{1}\left(\left[0, \Theta_{\gamma}\right]\right)}\right)\right)\left\|\kappa_{E}\right\|_{\mathbb{L}^{2}\left(\left[t_{\mathrm{I}}, \Theta_{\max }-\Theta_{\gamma}\right]\right)}
$$

By hypothesis $\kappa_{E} \in B_{1}$, inequality (29) implies that:

$$
\left|\mathcal{V}\left[\kappa_{E}\right](y)-\mathcal{V}\left[\kappa_{E}\right](x)\right|<\sqrt{|y-x|} \times\left(\|\gamma\|_{\mathbb{L}^{1}\left(\left[0, \Theta_{\gamma}\right]\right)}+|\alpha|\left(1+\|\gamma\|_{\mathbb{L}^{1}\left(\left[0, \Theta_{\gamma}\right]\right)}\right)\right)
$$

We say that $E_{1}$ is an equicontinuous subset of $\mathcal{C}_{c}\left(\left[t_{\mathrm{I}}, \Theta_{\max }\right]\right)$, if and only if, for every $\epsilon>0$ there exists $\eta>0$ depending on $\epsilon$ such that for every $x$ and $y$ in $\left[t_{I}, \Theta_{\max }\right]$ with $|x-y| \leq \eta$ implies that $\left|\mathcal{V}\left[\kappa_{E}\right](y)-\mathcal{V}\left[\kappa_{E}\right](x)\right|<\epsilon$. Given $\epsilon>0$ choosing $\eta$ such that: 


$$
\eta=\left(\frac{\epsilon}{\|\gamma\|_{\mathbb{L}^{1}\left(\left[0, \Theta_{\gamma}\right]\right)}+|\alpha|\left(1+\|\gamma\|_{\mathbb{L}^{1}\left(\left[0, \Theta_{\gamma}\right]\right)}\right)}\right)^{2},
$$

in order to get this equicontinuity property. According to (30) and (31), we get implication defined by relation (25). Consequently, $E_{1}$ is equicontinuous subset of $\mathcal{C}_{c}\left(\left[t_{\mathrm{I}}, \Theta_{\text {max }}\right]\right)$ allowing that $\mathcal{V}$ is compact in $\mathcal{C}_{c}\left(\left[t_{\mathrm{I}}, \Theta_{\max }\right]\right)$. Since operator $\tilde{\mathcal{V}}=i \circ \mathcal{V}$ is the composition of two compact operators which are the canonical injection $i$ defined from $\mathcal{C}_{c}\left(\left[t_{\mathrm{I}}, \Theta_{\text {max }}\right]\right)$ to $\mathbb{L}^{2}\left(\left[t_{\mathrm{I}}, \Theta_{\text {max }}\right]\right)$ and operator $\mathcal{V}, \tilde{\mathcal{V}}$ is compact on $\mathbb{L}^{2}\left(\left[t_{\mathrm{I}}, \Theta_{\max }\right]\right)$. From this, operator $\mathcal{L}$ is not compact on $\mathbb{L}^{2}\left(\left[t_{\mathrm{I}}, \Theta_{\max }\right]\right)$, completing the proof of the lemma. The mapping $\mathcal{L}$ is what we call a Hilbert-Schmidt operator.

Lemma 2.2. Linear operator $\mathcal{L}$ given by relation $(5)$ is not Fredholm operator.

Proof. Let be $E$ and $F$ be complex Hilbert space (infinite dimensional and separable) and let us recall that a Fredholm operator $T$ from $E$ to $F$ is bounded linear operator such that:

- $T$ and $T^{*}$ have finite dimensional null-spaces (or kernels);

- $T$ has closed image (so that $\left.\operatorname{Im}(T)=\left(\operatorname{ker}\left(T^{*}\right)\right)^{\perp}\right)$.

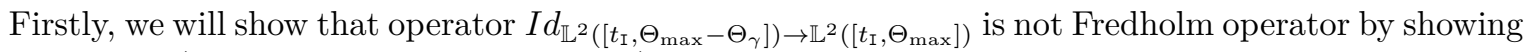

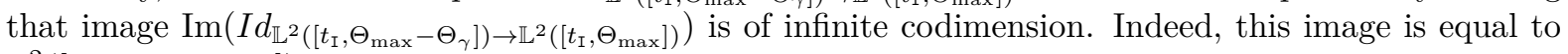
$\mathbb{L}^{2}\left(\left[t_{\mathrm{I}}, \Theta_{\max }-\Theta_{\gamma}\right]\right)$ :

$$
\operatorname{Im}\left(I_{\mathbb{L}^{2}\left(\left[t_{\mathrm{I}}, \Theta_{\max }-\Theta_{\gamma}\right]\right) \rightarrow \mathbb{L}^{2}\left(\left[t_{\mathrm{I}}, \Theta_{\max }\right]\right)}\right)=\mathbb{L}^{2}\left(\left[t_{\mathrm{I}}, \Theta_{\max }-\Theta_{\gamma}\right]\right) .
$$

From this, its complementary is defined as:

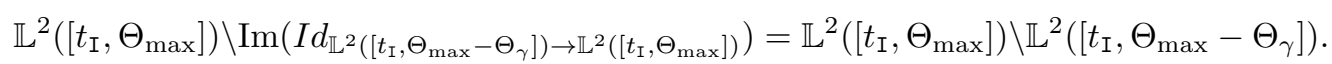

Since there are infinity functions which are in $\mathbb{L}^{2}\left(\left[t_{\mathrm{I}}, \Theta_{\max }\right]\right)$, and are not in $\mathbb{L}^{2}\left(\left[t_{\mathrm{I}}, \Theta_{\max }-\Theta_{\gamma}\right]\right)$, the com-

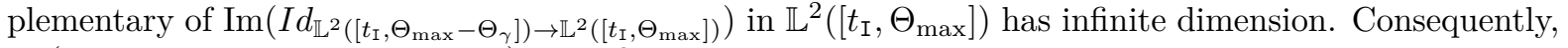

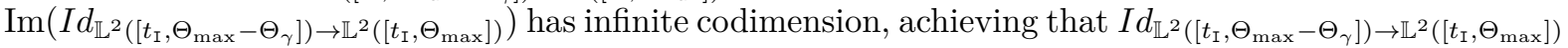
is not Fredholm operator.

Now, we use decomposition (13) in order to show that $\mathcal{L}$ is not Fredholm operator with using absurd reasoning. In order to emerge a contradiction, we use the fact that the sum of compact and Fredhom operators is Fredholm operator. Assuming that $\mathcal{L}$ is Fredholm operator. From this assumption and since we have shown in lemma 2.1 that $\tilde{\mathcal{V}}$ is compact, we obtain that $I d_{\mathbb{L}^{2}\left(\left[t_{\mathrm{I}}, \Theta_{\max }-\Theta_{\gamma}\right]\right) \rightarrow \mathbb{L}^{2}\left(\left[t_{\mathrm{I}}, \Theta_{\max }\right]\right)}$ is Fredholm operator, contradicting (33).

Lemma 2.3. Linear operator $\mathcal{D}$ acting on Initial Debt Repayment Density $\rho_{\mathcal{K}}^{\mathrm{I}} \in \mathbb{L}^{2}\left(\left[t_{\mathrm{I}}, \Theta_{\text {max }}\right]\right)$ defined as:

$$
\mathcal{D}\left[\rho_{\mathcal{K}}^{\mathrm{I}}\right](t)=-\alpha \int_{t}^{\Theta_{\max }} \rho_{\mathcal{K}}^{\mathrm{I}}(s) d s-\rho_{\mathcal{K}}^{\mathrm{I}}(t),
$$

is continuous and is not compact operator on $\mathbb{L}^{2}\left(\left[t_{\mathrm{I}}, \Theta_{\max }\right]\right)$. 
Proof. We begin by showing that operator $\mathcal{D}$ is well defined and continuous in $\mathbb{L}^{2}\left(\left[t_{\mathrm{I}}, \Theta_{\max }\right]\right)$. The triangle inequality is applied to operator $\mathcal{D}$ defined by (34) to get:

$$
\left\|\mathcal{D}\left[\rho_{\mathcal{K}}^{\mathrm{I}}\right]\right\|_{\mathbb{L}^{2}\left(\left[t_{\mathrm{I}}, \Theta_{\max }\right]\right)} \leq|\alpha| \times\left\|\int_{t}^{\Theta_{\max }} \rho_{\mathcal{K}}^{\mathrm{I}}(s) d s\right\|_{\mathbb{L}^{2}\left(\left[t_{\mathrm{I}}, \Theta_{\max }\right]\right)}+\left\|\rho_{\mathcal{K}}^{\mathrm{I}}\right\|_{\mathbb{L}^{2}\left(\left[t_{\mathrm{I}}, \Theta_{\max }\right]\right)} .
$$

Since we have $\left\|\int_{t}^{\Theta_{\max }} \rho_{\mathcal{K}}^{\mathrm{I}}(s) d s\right\|_{\mathbb{L}^{2}\left(\left[t_{\mathrm{I}}, \Theta_{\max }\right]\right)} \leq\left(\Theta_{\max }-t_{\mathrm{I}}\right) \times\left\|\rho_{\mathcal{K}}^{\mathrm{I}}\right\|_{\mathbb{L}^{2}\left(\left[t_{\mathrm{I}}, \Theta_{\max }\right]\right)}$ and according to (35), we have:

$$
\left\|\mathcal{D}\left[\rho_{\mathcal{K}}^{\mathrm{I}}\right]\right\|_{\mathbb{L}^{2}\left(\left[t_{\mathrm{I}}, \Theta_{\max }\right]\right)} \leq\left(|\alpha| \times\left(\Theta_{\max }-t_{\mathrm{I}}\right)+1\right) \times\left\|\rho_{\mathcal{K}}^{\mathrm{I}}\right\|_{\mathbb{L}^{2}\left(\left[t_{\mathrm{I}}, \Theta_{\max }\right]\right)} .
$$

Similarly as in lemma 2.1 , we will show that $\mathcal{D}$ is not compact on $\mathbb{L}^{2}\left(\left[t_{I}, \Theta_{\max }\right]\right)$. Decomposing operator $\mathcal{D}$ in following form:

$$
\mathcal{D}\left[\rho_{\mathcal{K}}^{\mathrm{I}}\right]+\tilde{\mathcal{W}}\left[\rho_{\mathcal{K}}^{\mathrm{I}}\right]=I d_{\mathbb{L}^{2}\left(\left[t_{\mathrm{I}}, \Theta_{\max }\right]\right)},
$$

where operator $\tilde{\mathcal{W}}: \mathbb{L}^{2}\left(\left[t_{\mathrm{I}}, \Theta_{\max }\right]\right) \rightarrow \mathbb{L}^{2}\left(\left[t_{\mathrm{I}}, \Theta_{\text {max }}\right]\right)$ is defined as operator acting on Initial Debt Repayment Density $\rho_{\mathcal{K}}^{\mathrm{I}}$ in form:

$$
\tilde{\mathcal{W}}\left[\rho_{\mathcal{K}}^{\mathrm{I}}\right](t)=\alpha \int_{t}^{\Theta_{\max }} \rho_{\mathcal{K}}^{\mathrm{I}}(s) d s .
$$

The aim of (37) is to use absurd reasoning in order to show that $\mathcal{D}$ is not compact. Next, defining operator $\mathcal{W}: \mathbb{L}^{2}\left(\left[t_{\mathrm{I}}, \Theta_{\text {max }}\right]\right) \rightarrow \mathcal{C}_{c}\left(\left[t_{\mathrm{I}}, \Theta_{\text {max }}\right]\right)$ as the considered operator $\tilde{\mathcal{W}}$ from $\mathbb{L}^{2}\left(\left[t_{\mathrm{I}}, \Theta_{\text {max }}\right]\right)$ to $\mathcal{C}_{c}\left(\left[t_{\mathrm{I}}, \Theta_{\max }\right]\right)$. Denoting $F_{1}$ by the image of open ball $B_{1}$

$$
F_{1}=\mathcal{W}\left(B_{1}\right) .
$$

We will verify that $F_{1}$ given by (39) satisfy the following conditions:

(i) $F_{1}$ is a bounded subset of $\mathcal{C}_{c}\left(\left[t_{\mathrm{I}}, \Theta_{\max }\right]\right)$;

(ii) $F_{1}$ is an equicontinuous subset of $\mathcal{C}_{c}\left(\left[t_{\mathrm{I}}, \Theta_{\text {max }}\right]\right)$.

In order to show first condition $(i)$, we use Cauchy-Schwarz inequality to get:

$$
\left|\mathcal{W}\left[\rho_{\mathcal{K}}^{\mathrm{I}}\right](t)\right| \leq|\alpha| \sqrt{\Theta_{\max }-t_{\mathrm{I}}}\left\|\rho_{\mathcal{K}}^{\mathrm{I}}\right\|_{\mathbb{L}^{2}\left(\left[t_{\mathrm{I}}, \Theta_{\max }\right]\right)} .
$$

And thus,

$$
\left\|\left|\mathcal{W} \|_{L^{\infty}\left(\left[t_{\mathrm{I}}, \Theta_{\max }\right]\right)} \leq\right| \alpha \mid \sqrt{\Theta_{\max }-t_{\mathrm{I}}},\right.
$$

which means that part $F_{1}$ is contained in closed ball of radius $|\alpha| \sqrt{\Theta_{\max }-t_{\mathrm{I}}}$ and origine center i.e. $F_{1} \subset \overline{B_{|\alpha| \sqrt{\Theta_{\max }-t_{\mathrm{I}}}}^{\prime}}$ achieving the proof $(i)$. In what to follows, we show second condition (ii). Using Cauchy-Schwarz inequality to get:

$$
\begin{aligned}
\left|\mathcal{W}\left[\rho_{\mathcal{K}}^{\mathrm{I}}\right](y)-\mathcal{W}\left[\rho_{\mathcal{K}}^{\mathrm{I}}\right](x)\right| & \leq|\alpha| \sqrt{|y-x|} \sqrt{\left|\int_{y}^{x}\left(\rho_{\mathcal{K}}^{\mathrm{I}}(s)\right)^{2} d s\right|}, \\
& \leq|\alpha| \sqrt{|y-x|}\left\|\rho_{\mathcal{K}}^{\mathrm{I}}\right\|_{\mathbb{L}^{2}\left(\left[t_{\mathrm{I}}, \Theta_{\max }\right]\right)} .
\end{aligned}
$$


For every $\epsilon>0$, we fix $\eta>0$ as:

$$
\eta=\left(\frac{\epsilon}{|\alpha|}\right)^{2},
$$

such that for every $x$ and $y$ in $\left[t_{\mathrm{I}}, \Theta_{\max }\right]$ with $|x-y| \leq \eta$, we get $\left|\mathcal{W}\left[\kappa_{E}\right](y)-\mathcal{W}\left[\kappa_{E}\right](x)\right|<\epsilon$ using inequality (42). Consequently, $F_{1}$ is equicontinuous subset of $\mathcal{C}_{c}\left(\left[t_{\mathrm{I}}, \Theta_{\max }\right]\right)$, achieving the proof $(i i)$. Ascoli theorem states that $\mathcal{W}$ is compact on $\mathcal{C}_{c}\left(\left[t_{\mathrm{I}}, \Theta_{\text {max }}\right]\right)$. Furthermore, operator $\tilde{\mathcal{W}}=i \circ \mathcal{W}$ is composed of two compact operators which are the canonical injection $i$ and operator $\mathcal{W}$, achieving that $\tilde{\mathcal{W}}$ is compact on $\mathbb{L}^{2}\left(\left[t_{\mathrm{I}}, \Theta_{\max }\right]\right)$. Then, $\mathcal{D}$ is not compact on $\mathbb{L}^{2}\left(\left[t_{\mathrm{I}}, \Theta_{\max }\right]\right)$, completing the proof of the lemma.

We can decompose the Algebraic Spending Density $\sigma$ defined in relation (1) as a sum of operators $\mathcal{L}$ and $\mathcal{D}$ :

$$
\sigma(t)=\mathcal{L}\left[\kappa_{E}\right](t)+\mathcal{D}\left[\rho_{\mathcal{K}}^{\mathrm{I}}\right](t) .
$$

Lemma 2.4. The singular point of function $1-\mathcal{F}(\gamma)$ is zero for any constant and affine density $\gamma$.

Proof. If Repayment Pattern Density $\gamma$ is a constant function given by:

$$
\gamma(t)=\frac{1}{\Theta_{\gamma}} \mathbb{1}_{\left[0, \Theta_{\gamma}\right]}(t),
$$

then we have:

$$
\forall \xi \in \mathbb{R}^{*}, 1-\mathcal{F}(\gamma)(\xi)=1-\frac{i}{\xi \Theta_{\gamma}}\left(e^{-i \xi \Theta_{\gamma}}-1\right)
$$

And thus,

$$
\begin{aligned}
\forall \xi \in \mathbb{R}^{*}, 1-\mathcal{F}(\gamma)(\xi)=0 & \Rightarrow \xi \Theta_{\gamma}=\sin \left(\xi \Theta_{\gamma}\right) \text { and } \cos \left(\xi \Theta_{\gamma}\right)=1, \\
& \Rightarrow\left(\xi \Theta_{\gamma}\right)^{2}+1=1, \\
& \Rightarrow \xi \Theta_{\gamma}=0 .
\end{aligned}
$$

Since real $\Theta_{\gamma}$ is positive, function $\xi \rightarrow 1-\mathcal{F}(\gamma)(\xi)$ is not zero function over $\mathbb{R}^{*}$. Conversely, if a real $\xi$ is zero, function $\xi \rightarrow 1-\mathcal{F}(\gamma)(\xi)$ is also a zero function. Indeed, the Fourier Transform of any density $\gamma$ at the origine is defined as:

$$
\mathcal{F}(\gamma)(0)=\int_{-\infty}^{+\infty} \gamma(t) d t
$$

Furthermore, since density $\gamma$ is with total mass which equals 1 , equality (48) implies that:

$$
\mathcal{F}(\gamma)(0)=1
$$

Now we will show that the singular point of function $1-\mathcal{F}(\gamma)$ is zero for an affine density $\gamma$ given by:

$$
\gamma(t)=\left(\frac{t}{\Theta_{\gamma}^{2}}+\frac{1}{2 \Theta_{\gamma}}\right) \mathbb{1}_{\left[0, \Theta_{\gamma}\right]}(t) .
$$

The integration by parts states that: 


$$
\forall \xi \in \mathbb{R}^{*}, 1-\mathcal{F}(\gamma)(\xi)=1-\left(\frac{1}{\left(\xi \Theta_{\gamma}\right)^{2}}+\frac{i}{2 \xi \Theta_{\gamma}}\right)\left(e^{-i \xi \Theta_{\gamma}}-1\right)-\frac{i}{\xi \Theta_{\gamma}} e^{-i \xi \Theta_{\gamma}} .
$$

From this, we get the following system of equations:

$$
\left\{\begin{array}{l}
\left(\cos \left(\xi \Theta_{\gamma}\right)-1\right)+\frac{3 \xi \Theta_{\gamma}}{2} \sin \left(\xi \Theta_{\gamma}\right)=\left(\xi \Theta_{\gamma}\right)^{2}, \\
-\sin \left(\xi \Theta_{\gamma}\right)+\xi \Theta_{\gamma} \cos \left(\xi \Theta_{\gamma}\right)+\frac{\xi \Theta_{\gamma}}{2}\left(\cos \left(\xi \Theta_{\gamma}\right)-1\right)=0 .
\end{array}\right.
$$

Hence,

$$
\xi \Theta_{\gamma}\left(1+\frac{3\left(\xi \Theta_{\gamma}\right)^{2}}{2}\right)-\left(1+\frac{9\left(\xi \Theta_{\gamma}\right)^{2}}{4}\right) \sin \left(\xi \Theta_{\gamma}\right)=0
$$

Figure 1 states that equation (53) doesn't have no solution on $\mathbb{R}^{*}$. Consequently, function $\xi \rightarrow 1-\mathcal{F}(\gamma)(\xi)$ is not a zero function over $\mathbb{R}^{*}$. Conversely, assuming that real $\xi$ is zero, we get:

$$
\begin{aligned}
1-\mathcal{F}(\gamma)(0) & =1-\int_{-\infty}^{+\infty} \gamma, \\
& =1-\frac{1}{\Theta_{\gamma}^{2}} \int_{0}^{\Theta_{\gamma}} t d t-\frac{1}{2 \Theta_{\gamma}} \int_{0}^{\Theta_{\gamma}} d t, \\
& =0 .
\end{aligned}
$$

We conclude that function $1-\mathcal{F}(\gamma)$ is zero at the origine for density $\gamma$ given by (45) (or by (50)). In what to follows, we will extend this conclusion for any affine density $\gamma$ given by:

$$
\gamma(t)=\left(c_{2} t+c_{1}\right) \mathbb{1}_{\left[0, \Theta_{\gamma}\right]}(t)
$$

where coefficients $c_{1}$ and $c_{2}$ satisfy:

$$
c_{1}+\frac{c_{2} \Theta_{\gamma}}{2}=\frac{1}{\Theta_{\gamma}} .
$$

We obtain using the integration by parts:

$$
\forall \xi \in \mathbb{R}^{*}, 1-\mathcal{F}(\gamma)(\xi)=1-c_{2}\left(\frac{e^{-i \xi \Theta_{\gamma}}-1}{\xi^{2}}+\frac{i \Theta_{\gamma} e^{-i \xi \Theta_{\gamma}}}{\xi}\right)-\frac{i c_{1}\left(e^{-i \xi \Theta_{\gamma}}-1\right)}{\xi}=0 .
$$

Next, by separating the real and the imaginary parts of function $1-\mathcal{F}(\gamma)$, we obtain the following system of equations:

$$
\left\{\begin{array}{c}
1-c_{2}\left(\frac{\cos \left(\xi \Theta_{\gamma}\right)-1}{\xi^{2}}+\frac{\Theta_{\gamma} \sin \left(\xi \Theta_{\gamma}\right)}{\xi}\right)-\frac{c_{1} \sin \left(\xi \Theta_{\gamma}\right)}{\xi}=0 \\
c_{2}\left(-\frac{\sin \left(\xi \Theta_{\gamma}\right)}{\xi^{2}}+\frac{\Theta_{\gamma} \cos \left(\xi \Theta_{\gamma}\right)}{\xi}\right)+\frac{c_{1}\left(\cos \left(\xi \Theta_{\gamma}\right)-1\right)}{\xi}=0
\end{array}\right.
$$

Let us check the consistency of the system of equations given by (58). Indeed, if coefficients $c_{1}$ and $c_{2}$ are respectively equal to $\frac{1}{2 \Theta_{\gamma}}$ and $\frac{1}{\Theta_{\gamma}^{2}}$, then we get equations defined by (52). Thanks to (58), we get the following equality: 


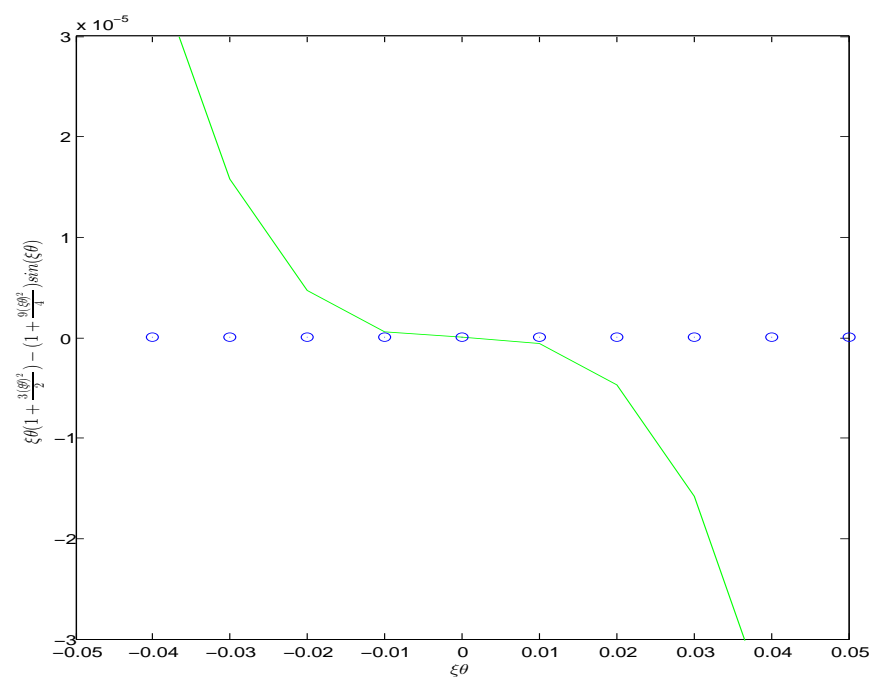

Figure 1: Graph of the function $\xi \Theta_{\gamma} \rightarrow \xi \Theta_{\gamma}\left(1+\frac{3\left(\xi \Theta_{\gamma}\right)^{2}}{2}\right)-\left(1+\frac{9\left(\xi \Theta_{\gamma}\right)^{2}}{4}\right) \sin \left(\xi \Theta_{\gamma}\right)$ over interval $[-0.05,0.05]$, showing that zero is its singular point.

$$
\frac{c_{2} \Theta_{\gamma}}{\xi}+\xi \Theta_{\gamma}+\frac{c_{1} \xi}{c_{2}}=\left(\frac{c_{2}}{\xi^{2}}+\Theta_{\gamma}\left(c_{2} \Theta_{\gamma}+c_{1}\right)+\frac{c_{1}\left(c_{2} \Theta_{\gamma}+c_{1}\right)}{c_{2}}\right) \sin \left(\xi \Theta_{\gamma}\right) .
$$

According to (58) and (59), we get:

$$
\frac{c_{2} \Theta_{\gamma}}{\xi}+\xi \Theta_{\gamma}+\frac{c_{1} \xi}{c_{2}}=\left(\frac{c_{2}}{\xi^{2}}+2+\frac{c_{1}^{2}}{c_{2}}\right) \sin \left(\xi \Theta_{\gamma}\right)
$$

We will show that equality (60) is consistent. Assuming that coefficients $c_{1}$ and $c_{2}$ are respectively equal to $\frac{1}{2 \Theta_{\gamma}}$ and $\frac{1}{\Theta_{\gamma}^{2}}$, we get equality defined by (53). Otherwise, equality (60) gives:

$$
\sin \left(\xi \Theta_{\gamma}\right)-\xi \Theta_{\gamma}\left(\frac{c_{2} \Theta_{\gamma}^{2}+\left(\xi \Theta_{\gamma}\right)^{2}\left(1+\frac{c_{1}}{c_{2} \Theta_{\gamma}}\right)}{c_{2} \Theta_{\gamma}^{2}+\left(\xi \Theta_{\gamma}\right)^{2}\left(2+\frac{c_{1}^{2}}{c_{2}}\right)}\right)=0 .
$$

Equalities (56) and (61) are multiplied by $\frac{c_{1}}{c_{2}}$ to give the following system of equations:

$$
\left\{\begin{array}{l}
\frac{c_{1}^{2}}{c_{2}}=-1+\frac{1}{c_{2} \Theta_{\gamma}^{2}}+\frac{c_{2} \Theta_{\gamma}^{2}}{4}, \\
\frac{c_{1}}{c_{2} \Theta_{\gamma}}=\frac{1}{c_{2} \Theta_{\gamma}^{2}}-\frac{1}{2} .
\end{array}\right.
$$


Replacing $\frac{c_{1}^{2}}{c_{2}}$ and $\frac{c_{1}}{c_{2} \Theta_{\gamma}}$ defined by (62) in equality (61), we obtain the following equality:

$$
\sin \left(\xi \Theta_{\gamma}\right)-\xi \Theta_{\gamma}\left(\frac{c_{2} \Theta_{\gamma}^{2}+\left(\xi \Theta_{\gamma}\right)^{2}\left(\frac{1}{2}+\frac{1}{c_{2} \Theta_{\gamma}^{2}}\right)}{c_{2} \Theta_{\gamma}^{2}+\left(\xi \Theta_{\gamma}\right)^{2}\left(1+\frac{1}{c_{2} \Theta_{\gamma}^{2}}+\frac{c_{2} \Theta_{\gamma}^{2}}{4}\right)}\right)=0
$$

As

$$
\forall \xi \in \mathbb{R}^{*},\left|\frac{\sin \left(\xi \Theta_{\gamma}\right)}{\xi \Theta_{\gamma}}\right| \leq 1,
$$

and according to (63), we obtain the following inequality:

$$
\frac{1}{2}+\frac{1}{c_{2} \Theta_{\gamma}^{2}} \leq 1+\frac{1}{c_{2} \Theta_{\gamma}^{2}}+\frac{c_{2} \Theta_{\gamma}^{2}}{4}
$$

which is simplified to give:

$$
-2 \leq c_{2} \Theta_{\gamma}^{2}
$$

Figure 2 shows that equation (63) doesn't have no solution on $\mathbb{R}^{*}$. Consequently, the proof of the lemma is achieved.

Many inverse problems in finance involve its study with regular singularities. For instance, paper [9] deals with inversing the differential operators on the half-line having a discontinuity in an interior. In the paper [12], several formally determined inverse problems are considered with recovering an important feature over singularities. For studying the inverse problem we agree that together with function $1-\mathcal{F}(\gamma)$ we suppose that zero is only its singularity for any density $\gamma$. This assumption gives a constructive procedure for the validity of the following theorem.

Theorem 2.5. If Repayment Pattern $\gamma$ satisfies relation (3) and following relation:

$$
\exists \epsilon>0, \frac{1}{1-\mathcal{F}(\gamma)}_{\mid]-\infty,-\epsilon[\cup] \epsilon,+\infty[} \in \mathbb{L}^{\infty}(\mathbb{R}),
$$

where $\mathcal{F}$ stands for the Fourier Transform Operator. And if Initial Debt Repayment Density $\rho_{\mathcal{K}}^{\mathrm{I}}$ is in $\mathbb{L}^{2}\left(\left[t_{\mathrm{I}}, \Theta_{\max }\right]\right)$, then for any Algebraic Spending Density $\sigma$ in $\mathbb{L}^{2}\left(\left[t_{\mathrm{I}}, \Theta_{\max }\right]\right)$ satisfies the following equality:

$$
\int_{t_{\mathrm{I}}}^{\Theta_{\max }}\left(\sigma(y)-\mathcal{D}\left[\rho_{\mathcal{K}}^{\mathrm{I}}\right](y)+\alpha \int_{t_{\mathrm{I}}}^{y}\left(\sigma(s)-\mathcal{D}\left[\rho_{\mathcal{K}}^{\mathrm{I}}\right](s)\right) \mathrm{e}^{\alpha(y-s)} d s\right) d y=0,
$$

there exists an unique Loan Density $\kappa_{E}$ stable in $\mathbb{L}^{2}\left(\left[t_{\mathrm{I}}, \Theta_{\max }-\Theta_{\gamma}\right]\right)$ which is given in terms of $\sigma$ by:

$$
\kappa_{E}=\mathcal{F}^{-1}\left(\frac{\mathcal{F}\left(\sigma-\mathcal{D}\left[\rho_{\mathcal{K}}^{\mathrm{I}}\right]+\alpha \int_{t_{\mathrm{I}}}^{\bullet}\left(\sigma(s)-\mathcal{D}\left[\rho_{\mathcal{K}}^{\mathrm{I}}\right](s)\right) \mathrm{e}^{\alpha(\bullet-s)} d s\right)}{1-\mathcal{F}(\gamma)}\right),
$$

where $\mathcal{F}$ stands for the Fourier Transform of function

$$
t \rightarrow \mathcal{F}\left(\left(\sigma-\mathcal{D}\left[\rho_{\mathcal{K}}^{\mathrm{I}}\right]\right)(t)+\alpha \int_{t_{\mathrm{I}}}^{t}\left(\sigma(s)-\mathcal{D}\left[\rho_{\mathcal{K}}^{\mathrm{I}}\right](s)\right) \mathrm{e}^{\alpha(t-s)} d s\right),
$$

and $\mathcal{F}^{-1}$ its inverse such that (44) holds. 


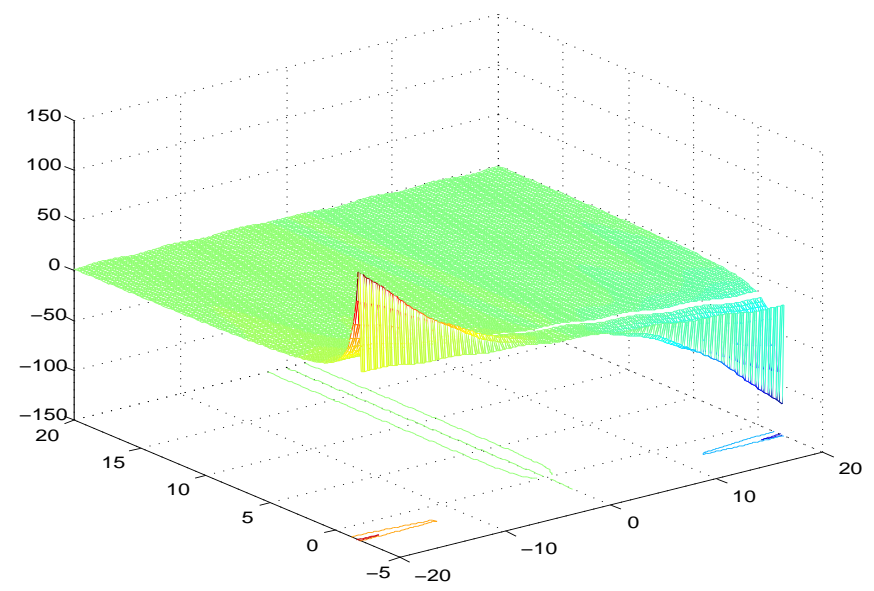

Figure 2: Graph of the function $\left(\xi \Theta_{\gamma}, c_{2} \Theta_{\gamma}^{2}\right) \rightarrow \sin \left(\xi \Theta_{\gamma}\right)-\xi \Theta_{\gamma}\left(\frac{c_{2} \Theta_{\gamma}^{2}+\left(\xi \Theta_{\gamma}\right)^{2}\left(\frac{1}{2}+\frac{1}{c_{2} \Theta_{\gamma}^{2}}\right)}{c_{2} \Theta_{\gamma}^{2}+\left(\xi \Theta_{\gamma}\right)^{2}\left(1+\frac{1}{c_{2} \Theta_{\gamma}^{2}}+\frac{c_{2} \Theta_{\gamma}^{2}}{4}\right)}\right)$ over interval $[-20,20] \times[-2,20]$, showing that zero is its singular point.

Proof of Theorem 2.5. We recall that we have shown in Lemma 3.5 in [7] that operator $\mathcal{L}$ given by relation (5) is a one-to-one application. From this, we obtain the uniqueness of $\kappa_{E}$.

If we assume that our noise (the error between measurement $\sigma_{2}^{I}$ and measurement $\sigma_{1}^{I}$ ) is small in the $\mathbb{L}^{2}$-norm, so that $\left\|\sigma_{2}^{I}-\sigma_{1}^{I}\right\|_{\mathbb{L}^{2}\left(\left[t_{\mathrm{I}}, \Theta_{\max }\right]\right)} \leq \delta$, and we are happy with a small error in the parameter in the $\mathbb{L}^{2}\left(\left[t_{\mathrm{I}}, \Theta_{\mathrm{max}}\right]\right)$ sense, then there is no problem. The reconstruction will be accurate in the sense that $\left\|\kappa_{E_{2}}-\kappa_{E_{1}}\right\|_{\mathbb{L}^{2}\left(\left[t_{\mathrm{I}}, \Theta_{\max }-\Theta_{\gamma}\right]\right)} \leq C_{\alpha}^{\gamma} \delta$, where $C_{\alpha}^{\gamma}$ is a real constant to be determined.

$$
\kappa_{E_{2}}-\kappa_{E_{1}}=\mathcal{F}^{-1}\left(\frac{\mathcal{F}\left(\sigma_{2}-\sigma_{1}+\alpha \int_{t_{\mathrm{I}}}^{\bullet}\left(\sigma_{2}-\sigma_{1}\right)(s) \mathrm{e}^{\alpha(\bullet-s)} d s\right)}{1-\mathcal{F}(\gamma)}\right) .
$$

Noticing that the Repayment Pattern Density $\gamma$ involved in this model satisfies:

$$
\int_{0}^{\Theta_{\gamma}} \gamma=1
$$

This equality means that $\mathcal{F}(\gamma)(\xi)$ converges to 1 when $\xi$ goes to 0 . Consequently, function $\xi \rightarrow \frac{1}{1-\mathcal{F}(\gamma)(\xi)}$ does not accept the upper bound. In other words, because of zero is only the singularity point of this 
function. We will show in some examples of density $\gamma$ that the upper bound is not reached. Setting density, i.e $\gamma=\frac{1}{\Theta_{\gamma}} \mathbb{1}_{\left[0, \Theta_{\gamma}\right]}$, we get:

$$
\left|\frac{1}{1-\mathcal{F}(\gamma)(\xi)}\right|=\frac{\left|\xi \Theta_{\gamma}\right|}{\sqrt{\left(\xi \Theta_{\gamma}-\sin \left(\xi \Theta_{\gamma}\right)\right)^{2}+\left(\cos \left(\xi \Theta_{\gamma}\right)-1\right)^{2}}} .
$$

Let us plot on $\mathbb{R}_{+}^{*}$ the function given by equality (73). This function is presented in Figure 3 showing that it does not attain the supremum on $\mathbb{R}^{*}$. For the same reason, we have:

$$
\frac{1}{|1-\mathcal{F}(\gamma)(\xi)|} \notin \mathbb{L}^{2}\left(\mathbb{R}^{*}\right)
$$

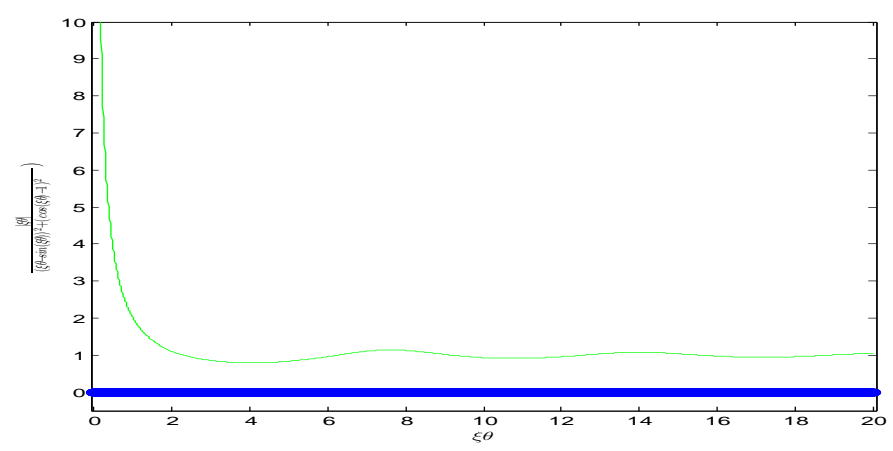

Figure 3: Graph of function $\xi \Theta_{\gamma} \rightarrow \frac{\left|\xi \Theta_{\gamma}\right|}{\sqrt{\left(\xi \Theta_{\gamma}-\sin \left(\xi \Theta_{\gamma}\right)\right)^{2}+\left(\cos \left(\xi \Theta_{\gamma}\right)-1\right)^{2}}}$ on $\mathbb{R}_{+}^{*}$ showing that the upper bound is not reached.

Now, we will show that density $\gamma$ satisfies relation (74). For instance, if density $\gamma$ is an affine function on its support $\left[0, \Theta_{\gamma}\right]$, i.e. $\gamma(t)=t \mathbb{1}_{[0, \sqrt{2}]}(t)$, we have:

$$
\left\|\frac{1}{|1-\mathcal{F}(\gamma)(\xi)|}\right\|_{\mathbb{L}^{2}(\mathbb{R})}^{2}=\frac{1}{\left(\left(1+\frac{1}{\xi^{2}}\right)-\left(\frac{\cos (\sqrt{2} \xi)}{\xi^{2}}+\frac{\sqrt{2} \sin (\sqrt{2} \xi)}{\xi}\right)\right)^{2}+\left(\frac{\sin (\sqrt{2} \xi)}{\xi^{2}}-\frac{\sqrt{2} \cos (\sqrt{2} \xi)}{\xi}\right)^{2}} .
$$

Matlab provides that the integral of targeted density is divergent as follows:

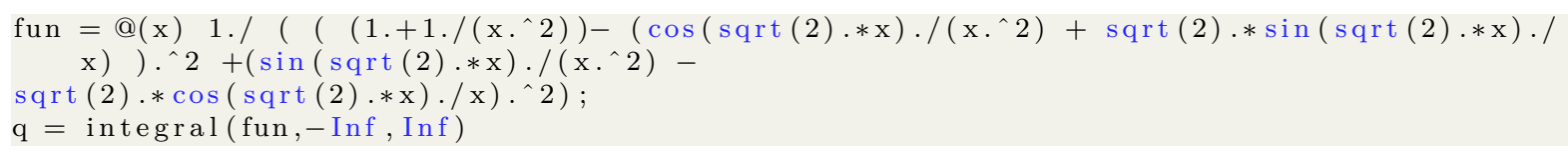

In these situations, we can not treat this inverse problem by bounding the term $\frac{1}{|1-\mathcal{F}(\gamma)(\xi)|}$ with respect 
to norm $\mathbb{L}^{2}(\mathbb{R})$ or $\mathbb{L}^{\infty}(\mathbb{R})$. For that, the density change is necessary in order to set up numerical stability problem. Assuming that there is a function $\phi \in \mathbb{L}^{2}\left(\left[t_{\mathbb{I}}, \Theta_{\max }\right]\right)$ satisfying $\mathcal{F}(\phi)(0)=0$ such that:

$$
\left|\frac{\mathcal{F}(\phi)(\xi)}{1-\mathcal{F}(\gamma)(\xi)}\right| \in \mathbb{L}^{2}(\mathbb{R})
$$

From this, there exists a constant $C^{\prime}$ such that:

$$
\left\|\left|\frac{\mathcal{F}(\phi)(\xi)}{1-\mathcal{F}(\gamma)(\xi)}\right|\right\|_{\mathbb{L}^{2}(\mathbb{R})} \leq C^{\prime}
$$

Since Inverse Fourier Transform $\mathcal{F}^{-1}$ preserves norm from $\mathbb{L}^{2}(\mathbb{R})$ to $\mathbb{L}^{2}\left(\left[t_{\mathrm{I}}, \Theta_{\max }\right]\right)$, we obtain from $(71)$ the following equality:

$$
\left\|\kappa_{E_{2}}-\kappa_{E_{1}}\right\|_{\mathbb{L}^{2}\left(\left[t_{\mathrm{I}}, \Theta_{\max }-\Theta_{\gamma}\right]\right)}=\left\|\frac{\mathcal{F}\left(\sigma_{2}-\sigma_{1}+\alpha \int_{t_{\mathrm{I}}}^{\bullet}\left(\sigma_{2}-\sigma_{1}\right)(s) \mathrm{e}^{\alpha(\bullet-s)} d s\right)}{\mathcal{F}(\phi)} \times \frac{\mathcal{F}(\phi)}{1-\mathcal{F}(\gamma)}\right\|_{\mathbb{L}^{2}(\mathbb{R})} .
$$

From this, we obtain:

$$
\begin{aligned}
\left\|\kappa_{E_{2}}-\kappa_{E_{1}}\right\|_{\mathbb{L}^{2}\left(\left[t_{\mathrm{I}}, \Theta_{\max }-\Theta_{\gamma}\right]\right)} & \leq\left\|\frac{\mathcal{F}\left(\sigma_{2}-\sigma_{1}+\alpha \int_{t_{\mathrm{I}}}^{\bullet}\left(\sigma_{2}-\sigma_{1}\right)(s) \mathrm{e}^{\alpha(\bullet-s)} d s\right)}{\mathcal{F}(\phi)}\right\|_{\mathbb{L}^{\infty}(\mathbb{R})}\left\|\frac{\mathcal{F}(\phi)}{1-\mathcal{F}(\gamma)}\right\|_{\mathbb{L}^{2}(\mathbb{R})}, \\
& \leq C^{\prime}\left\|\frac{\mathcal{F}\left(\sigma_{2}-\sigma_{1}\right)+\alpha \mathcal{F}\left(\int_{t_{\mathrm{I}}}^{\bullet}\left(\sigma_{2}-\sigma_{1}\right)(s) \mathrm{e}^{\alpha(\bullet-s)} d s\right)}{\mathcal{F}(\phi)}\right\|_{\mathbb{L}_{(\mathbb{R})}} .
\end{aligned}
$$

Defining densities $\sigma_{1}^{I}$ and $\sigma_{2}^{I}$ as:

$$
\sigma_{1}=\sigma_{1}^{I} \star \phi, \sigma_{2}=\sigma_{2}^{I} \star \phi .
$$

In which these densities $\sigma_{1}^{I}$ and $\sigma_{2}^{I}$ satisfy the following equality:

$$
\int_{t_{\mathrm{I}}}^{\Theta_{\max }}\left(\int_{t_{\mathrm{I}}}^{t}\left(\int_{t_{\mathrm{I}}}^{\Theta_{\max }}\left(\sigma_{2}^{I}-\sigma_{1}^{I}\right)(s-y) \phi(y) d y\right) \mathrm{e}^{\alpha(t-s)} d s\right) d t=0 .
$$

In the sequel, we use this definition (80) to get:

$$
\begin{aligned}
\left\|\frac{\mathcal{F}\left(\int_{t_{\mathrm{I}}}^{t}\left(\sigma_{2}-\sigma_{1}\right)(s) \mathrm{e}^{\alpha(t-s)} d s\right)}{\mathcal{F}(\phi)}\right\|_{\mathbb{L}^{\infty}(\mathbb{R})} & =\left\|\frac{\mathcal{F}\left(\int_{t_{\mathrm{I}}}^{t}\left(\left(\sigma_{2}^{I}-\sigma_{1}^{I}\right) \star \phi\right)(s) \mathrm{e}^{\alpha(t-s)} d s\right)}{\mathcal{F}(\phi)}\right\|_{\mathbb{L}^{\infty}(\mathbb{R})}, \\
& =\left\|\frac{\mathcal{F}\left(\int_{t_{\mathrm{I}}}^{t}\left(\int_{t_{\mathrm{I}}}^{\Theta_{\max }}\left(\sigma_{2}^{I}-\sigma_{1}^{I}\right)(s-y) \phi(y) d y\right) \mathrm{e}^{\alpha(t-s)} d s\right)}{\mathcal{F}(\phi)}\right\|_{\mathbb{L}^{\infty}(\mathbb{R})} .
\end{aligned}
$$


On other hand, we have:

$$
\begin{aligned}
& \left|\mathcal{F}\left(\int_{t_{\mathrm{I}}}^{t}\left(\int_{t_{\mathrm{I}}}^{\Theta_{\max }}\left(\sigma_{2}^{I}-\sigma_{1}^{I}\right)(s-y) \phi(y) d y\right) \mathrm{e}^{\alpha(t-s)} d s\right)(\xi)\right| \\
& \leq\left\|\int_{t_{\mathrm{I}}}^{t}\left(\int_{t_{\mathrm{I}}}^{\Theta_{\max }}\left(\sigma_{2}^{I}-\sigma_{1}^{I}\right)(s-y) \phi(y) d y\right) \mathrm{e}^{\alpha(t-s)} d s\right\|_{\mathbb{L}^{1}\left(\left[t_{\mathrm{I}}, \Theta_{\max }\right]\right)}, \\
& \leq \sup _{s \in\left[t_{\mathrm{I}}, \Theta_{\max }\right]}\left\{\mathrm{e}^{|\alpha|\left(\Theta_{\max }-s\right)}\right\}\left\|\int_{t_{\mathrm{I}}}^{\Theta_{\max }}\left(\int_{t_{\mathrm{I}}}^{\Theta_{\max }}\left(\sigma_{2}^{I}-\sigma_{1}^{I}\right)(s-y) \phi(y) d y\right) d s\right\|_{\mathbb{L}^{1}\left(\left[t_{\mathrm{I}}, \Theta_{\max }\right]\right)}, \\
& \leq \sup _{s \in\left[t_{\mathrm{I}}, \Theta_{\max }\right]}\left\{\mathrm{e}^{|\alpha|\left(\Theta_{\max }-s\right)}\right\}\left\|\int_{t_{\mathrm{I}}}^{\Theta_{\max }}\left(\int_{t_{\mathrm{I}}}^{\Theta_{\max }}\left(\sigma_{2}^{I}-\sigma_{1}^{I}\right)(s-y) \mathrm{e}^{+i \xi y} \phi(y) \mathrm{e}^{-i \xi y} d y\right) d s\right\|_{\mathbb{L}^{1}\left(\left[t_{\mathrm{I}}, \Theta_{\max }\right]\right)}, \\
& \leq \sup _{s \in\left[t_{\mathrm{I}}, \Theta_{\max }\right]}\left\{\mathrm{e}^{|\alpha|\left(\Theta_{\max }-s\right)}\right\} \int_{t_{\mathrm{I}}}^{\Theta_{\max }}\left|\int_{t_{\mathrm{I}}}^{\Theta_{\max }}\left(\int_{t_{\mathrm{I}}}^{\Theta_{\max }}\left(\sigma_{2}^{I}-\sigma_{1}^{I}\right)(s-y) \mathrm{e}^{+i \xi y} \phi(y) \mathrm{e}^{-i \xi y} d y\right) d s\right| d t, \\
& \leq\left(\Theta_{\max }-t_{\mathrm{I}}\right) \sup _{s \in\left[t_{\mathrm{I}}, \Theta_{\max }\right]}\left\{\mathrm{e}^{|\alpha|\left(\Theta_{\max }-s\right)}\right\}\left|\int_{t_{\mathrm{I}}}^{\Theta_{\max }}\left(\int_{t_{\mathrm{I}}}^{\Theta_{\max }}\left(\sigma_{2}^{I}-\sigma_{1}^{I}\right)(s-y) \mathrm{e}^{+i \xi y} \phi(y) \mathrm{e}^{-i \xi y} d y\right) d s\right|, \\
& \leq\left(\Theta_{\max }-t_{\mathrm{I}}\right) \sup _{s \in\left[t_{\mathrm{I}}, \Theta_{\max }\right]}\left\{\mathrm{e}^{|\alpha|\left(\Theta_{\max }-s\right)}\right\}\left\|\left|\left(\sigma_{2}^{I}-\sigma_{1}^{I}\right) \mathrm{e}^{+i \xi y}\right|\right\|_{\mathbb{L}^{\infty}\left(\left[t_{\mathrm{I}}, \Theta_{\max }\right]\right)}\left|\int_{t_{\mathrm{I}}}^{\Theta_{\max }}\left(\int_{t_{\mathrm{I}}}^{\Theta_{\max }} \phi(y) \mathrm{e}^{-i \xi y} d y\right) d s\right|, \\
& \leq\left(\Theta_{\max }-t_{\mathrm{I}}\right) \sup _{s \in\left[t_{\mathrm{I}}, \Theta_{\max }\right]}\left\{\mathrm{e}^{|\alpha|\left(\Theta_{\max }-s\right)}\right\}\left\|\left|\left(\sigma_{2}^{I}-\sigma_{1}^{I}\right) \mathrm{e}^{+i \xi y}\right|\right\|_{\mathbb{L}^{\infty}\left(\left[t_{\mathrm{I}}, \Theta_{\max }\right]\right)} \int_{t_{\mathrm{I}}}^{\Theta_{\max }}\left|\int_{t_{\mathrm{I}}}^{\Theta_{\max }} \phi(y) \mathrm{e}^{-i \xi y} d y\right| d s \\
& \leq\left(\Theta_{\max }-t_{\mathrm{I}}\right)^{2} \sup _{s \in\left[t_{\mathrm{I}}, \Theta_{\max }\right]}\left\{\mathrm{e}^{|\alpha|\left(\Theta_{\max }-s\right)}\right\}\left\|\left|\left(\sigma_{2}^{I}-\sigma_{1}^{I}\right) \mathrm{e}^{+i \xi y}\right|\right\|_{\mathbb{L}^{\infty}\left(\left[t_{\mathrm{I}}, \Theta_{\max }\right]\right)}\left|\int_{t_{\mathrm{I}}}^{\Theta_{\max }} \phi(y) \mathrm{e}^{-i \xi y} d y\right|, \\
& \leq\left(\Theta_{\max }-t_{\mathrm{I}}\right)^{2} \sup _{s \in\left[t_{\mathrm{I}}, \Theta_{\max }\right]}\left\{\mathrm{e}^{|\alpha|\left(\Theta_{\max }-s\right)}\right\}|\mathcal{F}(\phi)(\xi)|\left\|\sigma_{2}^{I}-\sigma_{1}^{I}\right\|_{\mathbb{L}^{\infty}\left(\left[t_{\mathrm{I}}, \Theta_{\max }\right]\right)} .
\end{aligned}
$$

Finally, equality (83) gives:

$$
\begin{aligned}
\left|\frac{\mathcal{F}\left(\int_{t_{\mathrm{I}}}^{t}\left(\int_{t_{\mathrm{I}}}^{\Theta_{\max }}\left(\sigma_{2}^{I}-\sigma_{1}^{I}\right)(s-y) \phi(y) d y\right) \mathrm{e}^{\alpha(t-s)} d s\right)(\xi)}{\mathcal{F}(\phi)(\xi)}\right| \leq\left(\Theta_{\max }-t_{\mathrm{I}}\right)^{2} \sup _{s \in\left[t_{\mathrm{I}}, \Theta_{\max }\right]}\left\{\mathrm{e}^{|\alpha|\left(\Theta_{\max }-s\right)}\right\} & \left\|\sigma_{2}^{I}-\sigma_{1}^{I}\right\|_{\mathbb{L}^{\infty}\left(\left[t_{\mathrm{I}}, \Theta_{\max }\right]\right)} .
\end{aligned}
$$

In order to obtain a consistent stability, we impose on density $\sigma_{2}^{I}-\sigma_{1}^{I}$ the following inequality:

$$
\left\|\sigma_{2}^{I}-\sigma_{1}^{I}\right\|_{\mathbb{L}^{\infty}\left(\left[t_{I}, \Theta_{\max }\right]\right)} \leq\left\|\sigma_{2}^{I}-\sigma_{1}^{I}\right\|_{\mathbb{L}^{2}\left(\left[t_{I}, \Theta_{\max }\right]\right)} .
$$

According to equalities (84) and (85), we get:

$$
\left\|\frac{\mathcal{F}\left(\int_{t_{\mathrm{I}}}^{t}\left(\int_{t_{\mathrm{I}}}^{\Theta_{\max }}\left(\sigma_{2}^{I}-\sigma_{1}^{I}\right)(s-y) \phi(y) d y\right) \mathrm{e}^{\alpha(t-s)} d s\right)}{\mathcal{F}(\phi)}\right\|_{\mathbb{L}^{\infty}(\mathbb{R})} \leq\left(\Theta_{\max }-t_{\mathrm{I}}\right)^{2} \sup _{s \in\left[t_{\mathrm{I}}, \Theta_{\max }\right]}\left\{\mathrm{e}^{|\alpha|\left(\Theta_{\max }-s\right)}\right\}
$$

Using definition (80) of densities $\sigma_{2}^{I}$ et $\sigma_{1}^{I}$ coupled with convolution operator to get: 


$$
\begin{aligned}
\left\|\frac{\mathcal{F}\left(\sigma_{2}-\sigma_{1}\right)}{\mathcal{F}(\phi)}\right\|_{\mathbb{L}^{\infty}(\mathbb{R})} \leq\left\|\mathcal{F}\left(\sigma_{2}^{I}-\sigma_{1}^{I}\right)\right\|_{\mathbb{L}^{\infty}(\mathbb{R})}, \\
\leq\left\|\sigma_{2}^{I}-\sigma_{1}^{I}\right\|_{\mathbb{L}^{1}\left(\left[t_{\mathrm{I}}, \Theta_{\max }\right]\right)} .
\end{aligned}
$$

Using Cauchy-Schwarz inequality:

$$
\left\|\sigma_{2}^{I}-\sigma_{1}^{I}\right\|_{\mathbb{L}^{1}\left(\left[t_{\mathrm{I}}, \Theta_{\max }\right]\right)} \leq \sqrt{\Theta_{\max }-t_{\mathrm{I}}} \times\left\|\sigma_{2}^{I}-\sigma_{1}^{I}\right\|_{\mathbb{L}^{2}\left(\left[t_{\mathrm{I}}, \Theta_{\max }\right]\right)} .
$$

Consequently, we get:

$$
\begin{gathered}
\left\|\kappa_{E_{2}}-\kappa_{E_{1}}\right\|_{\mathbb{L}^{2}\left(\left[t_{\mathrm{I}}, \Theta_{\max }-\Theta_{\gamma}\right]\right)} \leq C^{\prime}\left(\sqrt{\Theta_{\max }-t_{\mathrm{I}}}+|\alpha|\left(\Theta_{\max }-t_{\mathrm{I}}\right)^{2} \mathrm{e}^{|\alpha|\left(\Theta_{\max }-t_{\mathrm{I}}\right)}\right) \\
\left\|\sigma_{2}^{I}-\sigma_{1}^{I}\right\|_{\mathbb{L}^{2}\left(\left[t_{\mathrm{I}}, \Theta_{\max }\right]\right)} .
\end{gathered}
$$

\section{Inverse problem of the model in $\mathcal{M}\left(\left[t_{\mathrm{I}}, \Theta_{\max }\right]\right)$}

The aim of this section is to study the inverse problem in measure space. Denoting $\mathcal{M}\left(\left[t_{\mathrm{I}}, \Theta_{\max }\right]\right)$ the Radon measure space which is a continuous and linear form acting on continuous functions space $\mathcal{C}_{c}\left(\left[t_{\mathrm{I}}, \Theta_{\max }\right]\right)$ defined over a time interval $\left[t_{\mathrm{I}}, \Theta_{\max }\right]$. The usual norm on $\mathcal{M}\left(\left[t_{\mathrm{I}}, \Theta_{\max }\right]\right)$ is:

$$
\|\mu\|_{\mathcal{M}\left(\left(t_{\mathrm{I}}, \Theta_{\max }\right)\right)}=\sup _{\psi \in \mathcal{C}_{c}\left(\left[t_{\mathrm{I}}, \Theta_{\max }\right]\right), \psi \neq 0}\left\{\frac{|\langle\mu, \psi\rangle|}{\|\psi\|_{L^{\infty}\left(\left[t_{\mathrm{I}}, \Theta_{\max }\right]\right)}}\right\}
$$

where \|\|$_{L^{\infty}\left(\left[t_{\mathrm{I}}, \Theta_{\max }\right]\right)}$ is the usual norm on $\mathcal{C}_{c}\left(\left[t_{\mathrm{I}}, \Theta_{\max }\right]\right)$ defined by $(17)$. We set the Repayment Pattern Measure $\tilde{\gamma}$ such that:

$$
\tilde{\gamma} \in \mathcal{M}\left(\left[0, \Theta_{\gamma}\right]\right),
$$

where $\Theta_{\gamma}$ is positive number satisfying relation (4). By relation (91), the support of convolution of two compactly supported measures $\tilde{\kappa}_{E}$ in $\left[t_{\mathrm{I}}, \Theta_{\max }-\Theta_{\gamma}\right]$ and $\tilde{\gamma}$ in $\left[0, \Theta_{\gamma}\right]$ is included in $\left[t_{\mathrm{I}}, \Theta_{\max }\right]$. Indeed, formally:

$$
\operatorname{Supp}\left(\tilde{\kappa}_{E} \star \tilde{\gamma}\right) \subset \operatorname{Supp}\left(\tilde{\kappa}_{E}\right)+\operatorname{Supp}(\tilde{\gamma}) .
$$

Let $\mathcal{L}_{1}$ be a linear operator defined from $\mathcal{M}\left(\left[t_{\mathrm{I}}, \Theta_{\max }-\Theta_{\gamma}\right]\right)$ to $\mathcal{M}\left(\left[t_{\mathrm{I}}, \Theta_{\max }\right]\right)$ acting on Loan Measure $\tilde{\kappa}_{E}$ by

$$
\mathcal{L}_{1}\left[\tilde{\kappa}_{E}\right]=\tilde{\kappa}_{E}-\tilde{\kappa}_{E} \star \tilde{\gamma}-\alpha\left\langle\tilde{\kappa}_{E}-\tilde{\kappa}_{E} \star \tilde{\gamma}, \mathbb{1}_{\mid\left[t_{I}, t\right]}\right\rangle d t .
$$

Let $\mathcal{D}_{1}$ be an operator defined in $\mathcal{M}\left(\left[t_{\mathrm{I}}, \Theta_{\text {max }}\right]\right)$ acting on Initial Debt Repayment Measure $\tilde{\rho}_{\mathcal{K}}^{\mathrm{I}}$ by

$$
\mathcal{D}_{1}\left[\tilde{\rho}_{\mathcal{K}}^{\mathrm{I}}\right]=-\alpha\left\langle\tilde{\rho}_{\mathcal{K}}^{\mathrm{I}}, \mathbb{1}_{\mid\left[t, \Theta_{\max }[\right.}\right\rangle d t-\tilde{\rho}_{\mathcal{K}}^{\mathrm{I}} .
$$

Algebraic Spending Measure $\tilde{\sigma}$ is defined such that the difference between spendings and incomes required to satisfy the current needs. Measure $\tilde{\sigma}$ is decomposed as a sum of operators $\mathcal{L}_{1}$ and $\mathcal{D}_{1}$ given by relations (93) and (94), respectively:

$$
\tilde{\sigma}=\mathcal{L}_{1}\left[\tilde{\kappa}_{E}\right]+\mathcal{D}_{1}\left[\tilde{\rho}_{\mathcal{K}}^{\mathrm{I}}\right] .
$$


Theorem 3.1. If Repayment Pattern Measure $\tilde{\gamma}$ is satisfying relation (91) and following relation

$$
\frac{1}{1-\mathcal{F}(\tilde{\gamma})_{\mid]-\infty,-\epsilon[\cup] \epsilon,+\infty[}} \in \mathbb{L}^{\infty}(\mathbb{R})
$$

for any positive real $\epsilon$ and if Loan Measure $\tilde{\kappa}_{E}$ exists in $\mathcal{M}\left(\left[t_{\mathrm{I}}, \Theta_{\max }-\Theta_{\gamma}\right]\right)$ for any Initial Debt Repayment Measure $\tilde{\rho}_{\mathcal{K}}^{\mathrm{I}}$ and for any Algebraic Spending Measure $\tilde{\sigma}$ in $\mathcal{M}\left(\left[t_{\mathrm{I}}, \Theta_{\max }\right]\right)$ satisfying the following equality:

$$
\tilde{\kappa}_{E}=\mathcal{F}^{-1}\left(\frac{\mathcal{F}\left(\tilde{\sigma}-\mathcal{D}_{1}\left[\tilde{\rho}_{\mathcal{K}}^{\mathrm{I}}\right]+\alpha \tilde{\mathrm{e}}_{\alpha}\left\langle\tilde{\sigma}-\mathcal{D}_{1}\left[\tilde{\rho}_{\mathcal{K}}^{\mathrm{I}}\right], \mathrm{e}_{-\alpha \mid\left[t_{\mathrm{I}}, t[\right.}\right\rangle\right)}{1-\mathcal{F}(\tilde{\gamma})}\right)
$$

where $\langle.,$.$\rangle stands for the duality bracket of measure space \mathcal{M}\left(\left[t_{\mathrm{I}}, \Theta_{\max }\right]\right)$ with space of functions containing piecewise continuous functions. Then, Loan Measure $\tilde{\kappa}_{E}$ is unique and stable in $\mathcal{M}\left(\left[t_{\mathrm{I}}, \Theta_{\max }-\Theta_{\gamma}\right]\right)$.

Proof. Notice that since the inverse Fourier transform $\mathcal{F}^{-1}$ is not surjective from $\mathcal{M}(\mathbb{R})$ to $\mathcal{M}\left(\left[t_{\mathrm{I}}, \Theta_{\max }\right]\right)$, the solution $\tilde{\kappa}_{E}$ doesn't exist for the inverse problem. Moreover, if Loan Measure $\tilde{\kappa}_{E}$ is supposed satisfying relation $(97)$, then it is unique due to the injectivity of operator $\mathcal{L}_{1}$ (see Lemma 3.4 in [7]).

Now we will show that solution Loan Measure $\tilde{\kappa}_{E}$ is stable. Definition (93) of operator $\mathcal{L}_{1}$ gives that for any two Loan Densities $\tilde{\kappa}_{E_{1}}$ and $\tilde{\kappa}_{E_{2}}$ the following equality:

$$
\tilde{\kappa}_{E_{2}}-\tilde{\kappa}_{E_{1}}=\mathcal{F}^{-1}\left(\frac{\mathcal{F}\left(\tilde{\sigma}_{2}-\tilde{\sigma}_{1}+\alpha \tilde{\mathrm{e}}_{\alpha}\left\langle\tilde{\sigma}_{2}-\tilde{\sigma}_{1}, \mathrm{e}_{-\alpha \mid\left[t_{\mathrm{I}}, t[\right.}\right\rangle\right)}{1-\mathcal{F}(\tilde{\gamma})}\right) .
$$

The usual Radon norm on $\mathcal{M}\left(\left[t_{\mathrm{I}}, \Theta_{\max }-\Theta_{\gamma}\right]\right)$ of $\tilde{\kappa}_{E_{2}}-\tilde{\kappa}_{E_{1}}$ is defined by:

$$
\left\|\tilde{\kappa}_{E_{2}}-\tilde{\kappa}_{E_{1}}\right\|_{\mathcal{M}\left(\left[t_{\mathrm{I}}, \Theta_{\max }-\Theta_{\gamma}\right]\right)}=\sup _{\phi \in \mathcal{C}_{c}\left(\left[t_{\mathrm{I}}, \Theta_{\max }-\Theta_{\gamma}\right]\right), \phi \neq 0}\left\{\frac{\left|\left\langle\tilde{\kappa}_{E_{2}}-\tilde{\kappa}_{E_{1}}, \phi\right\rangle\right|}{\|\phi\|_{L^{\infty}\left(\left[t_{\mathrm{I}}, \Theta_{\max }-\Theta_{\gamma}\right]\right)}}\right\} .
$$

Replacing measure $\tilde{\kappa}_{E_{2}}-\tilde{\kappa}_{E_{1}}$ given by (98) in right equality (99) yield the following equality:

$$
\begin{aligned}
\left\|\tilde{\kappa}_{E_{2}}-\tilde{\kappa}_{E_{1}}\right\|_{\mathcal{M}\left(\left[t_{\mathrm{I}}, \Theta_{\max }-\Theta_{\gamma}\right]\right)} & =\frac{|\mathcal{F}(\phi)|}{|1-\mathcal{F}(\tilde{\gamma})|} \\
& \sup _{\psi \in \mathcal{C}_{c}\left(\left[t_{\mathrm{I}}, \Theta_{\max }-\Theta_{\gamma}\right]\right), \psi \neq 0}\left\{\frac{\left|\left\langle\mathcal{F}\left(\tilde{\sigma}_{2}-\tilde{\sigma}_{1}+\alpha \tilde{\mathrm{e}}_{\alpha}\left\langle\tilde{\sigma}_{2}-\tilde{\sigma}_{1}, \mathrm{e}_{-\alpha \mid\left[t_{\mathrm{I}}, t[\right.}\right\rangle\right), \mathcal{F}(\psi)\right\rangle\right|}{\left.|\mathcal{F}(\phi)| \times\|\psi\|_{L^{\infty}\left(\left[t_{\mathrm{I}}, \Theta_{\max }-\Theta_{\gamma}\right]\right)}\right\} .}\right.
\end{aligned}
$$

Since function $\psi$ having support in $\left[t_{\mathrm{I}}, \Theta_{\max }-\Theta_{\gamma}\right]$, there exists a constant $C$ such as:

$$
\frac{|\mathcal{F}(\psi)|}{\|\psi\|_{L^{\infty}\left(\left[t_{\mathrm{I}}, \Theta_{\max }-\Theta_{\gamma}\right]\right)}} \leq C
$$

According to equalities (100) and (101), we obtain the following inequality:

$$
\begin{aligned}
& \left\|\tilde{\kappa}_{E_{2}}-\tilde{\kappa}_{E_{1}}\right\|_{\mathcal{M}\left(\left[t_{\mathrm{I}}, \Theta_{\max }-\Theta_{\gamma}\right]\right)} \leq C \frac{|\mathcal{F}(\phi)|}{|1-\mathcal{F}(\tilde{\gamma})|} \\
& \sup _{\psi \in \mathcal{C}_{c}\left(\left[t_{\mathrm{I}}, \Theta_{\max }-\Theta_{\gamma}\right]\right), \psi \neq 0}\left\{\left|\frac{\mathcal{F}\left(\tilde{\sigma}_{2}-\tilde{\sigma}_{1}+\alpha \tilde{\mathrm{e}}_{\alpha}\left\langle\tilde{\sigma}_{2}-\tilde{\sigma}_{1}, \mathrm{e}_{-\alpha \mid\left[t_{\mathrm{I}}, t[\right.}\right\rangle\right)}{\mathcal{F}(\phi)}\right|\right\} .
\end{aligned}
$$


Similary to the Theorem 2.5, we make the same assumptions in order to establish the stability to new modified Algebraic Spending Measures $\tilde{\sigma}_{1}^{I}$ and $\tilde{\sigma}_{2}^{I}$ (see definition (80)). As we have set up in equality (86), we obtain in the same way:

$$
\begin{aligned}
\left|\frac{\mathcal{F}\left(\tilde{\sigma}_{2}-\tilde{\sigma}_{1}+\alpha \tilde{\mathrm{e}}_{\alpha}\left\langle\tilde{\sigma}_{2}-\tilde{\sigma}_{1}, \mathrm{e}_{-\alpha \mid\left[t_{\mathrm{I}}, t \mid\right.}\right\rangle\right)}{\mathcal{F}(\phi)}\right| \leq\left(\Theta_{\max }-t_{\mathrm{I}}\right)^{2} \sup _{s \in\left[t_{\mathrm{I}}, \Theta_{\max }\right]}\left\{\mathrm{e}^{|\alpha|\left(\Theta_{\max }-s\right)}\right\} \\
\left\|\tilde{\sigma}_{2}^{I}-\tilde{\sigma}_{1}^{I}\right\|_{\mathcal{M}\left(\left[t_{\mathrm{I}}, \Theta_{\max }\right]\right) .}
\end{aligned}
$$

Consequently,

$$
\left\|\tilde{\kappa}_{E_{2}}-\tilde{\kappa}_{E_{1}}\right\|_{\mathcal{M}\left(\left[t_{I}, \Theta_{\max }-\Theta_{\gamma}\right]\right)} \leq C \times C^{\prime} \times\left(\Theta_{\max }-t_{\mathrm{I}}\right)^{2} \mathrm{e}^{|\alpha|\left(\Theta_{\max }-t_{\mathrm{I}}\right)}\left\|\tilde{\sigma}_{2}^{I}-\tilde{\sigma}_{1}^{I}\right\|_{\mathcal{M}\left(\left[t_{\mathrm{I}}, \Theta_{\max }\right]\right)},
$$

where $C^{\prime}$ is defined as (77).

\section{Conflict of Interests}

The authors declare that there is no conflict of interests.

\section{References}

[1] HP Baltes. Progress in inverse optical problems. In Inverse Scattering Problems in Optics, pages 1-13. Springer, 1980.

[2] Ronald I Becker. Periodic solutions of semilinear equations of evolution of compact type. Journal of Mathematical Analysis and Applications, 82(1):33-48, 1981.

[3] M Cecchi, M Furi, and M Marini. On continuity and compactness of some nonlinear operators associated with differential equations in noncompact intervals. Nonlinear Analysis: Theory, Methods ES Applications, 9(2):171-180, 1985.

[4] Tarik Chakkour. Some notes about the continuous-in-time financial model. Abstract and Applied Analysis, 2017, 2017.

[5] Tarik Chakkour. Some inverse problem remarks of a continuous-in-time financial model in $l 1$ ([ti, $\theta$ max]). Results in Applied Mathematics (Under Review), 2019.

[6] Tarik Chakkour. Implementing some mathematical operators for a continuous-in-time financial model. Engineering Mathematics Letters, Vol. 2017, Article ID 2.

[7] Tarik Chakkour and Emmanuel Frénod. Inverse problem and concentration method of a continuousin-time financial model. International Journal of Financial Engineering, 3(02):1650016, 2016.

[8] M De la Sen. Stability of impulsive time-varying systems and compactness of the operators mapping the input space into the state and output spaces. Journal of Mathematical Analysis and Applications, $321(2): 621-650,2006$.

[9] Gerhard Freiling and Vjacheslav Yurko. Inverse spectral problems for singular non-selfadjoint differential operators with discontinuities in an interior point. Inverse Problems, 18(3):757, 2002.

[10] Emmanuel Frenod and Tarik Chakkour. A continuous-in-time financial model. Mathematical Finance Letters, Vol. 2016, Article ID 2.

[11] J Hadamard. Lectures on the cauchy problem in linear partial differential equations, 1923.

[12] Ziqi Sun and Gunther Uhlmann. Recovery of singularities for formally determined inverse problems. Communications in Mathematical Physics, 153(3):431-445, 1993.

[13] Andrey N Tikonov and Vasily Y Arsenin. Solutions of ill-posed problems. New York: Winston, 1977. 